\title{
Microscopía holográfica digital sin lentes con resolución micrométrica y fuentes multiespectrales
}

\author{
Jorge Garcia-Sucerquia \\ Universidad Nacional de Colombia, Escuela de Física, Medellín, Colombia
}

\begin{abstract}
Resumen
Se presenta en este trabajo la microscopía holográfica digital sin lentes (MHDSL) como una metodología de formación de imágenes con resolución micrométrica sin el uso de lentes y el uso de fuentes multiespectrales. Los principios y desarrollos recientes en el dominio de esta metodología de microscopía son expuestos desde la perspectiva de los aportes realizados por nuestro grupo. MHDSL operando a color y con láseres de femto segundos son los avances resaltados en este trabajo. Los efectos de la coherencia espacial y temporal de la fuente de iluminación en el desempeño del MHDSL son analizados por medio de la comparación de las imágenes reconstruidas del microscopio usando un LED o un láser mode-locked Ti:Sa laser de 12 fs. Una sección de la cabeza de una mosca Drosophila melanogaster se utiliza como muestra con una compleja estructura interna.

Palabras clave: microscopía holográfica digital sin lentes, láser de femto segundos, Drosophila melanogaster, coherencia.

Digital lensless holographic microscopy with micrometric resolution and multispectral sources

Abstract

Digital lensless holographic microscopy (DLHM) as an imaging architecture with micrometer spatial resolution and operating with multispectral sources is shown. The principles and recent advances in the realm of this technology of microscopy are presented from the point of view of the contributions of our group. DLHM operating a full color and with femto-second lasers are the advances highlighted in this work. The effects of the spatial and the temporal coherence of the light source on the performance of the DLHM are analyzed by comparing the reconstructed images of the microscope by using a LED and a 12 fs mode-locked Ti:Sa laser. A section of the head of a Drosophila melanogaster fly is utilized as a sample with complex internal structure.
\end{abstract}

Key words: Digital lensless holographic microscopy, femto second laser, Drosophila melanogaster, coherence.

\section{Introducción}

La holografía es una técnica de formación de imágenes en dos pasos que permite recuperar la amplitud y la fase del frente de una onda llamada objeto (Goodman, 2005). En un primer paso, denominado registro, se almacena el patrón de interferencia entre la onda objeto y una onda de referencia de la cual se conoce su amplitud y fase. A partir de este registro de intensidad, denominado holograma, se puede recuperar la amplitud compleja de la onda objeto en la segunda etapa de la holografía, denominada reconstrucción. En su concepción original de configuración en línea (Gabor, 1948; Gabor, 1949; Gabor, 1951), la holografía no fue una técnica con gran acogida debido a las limitaciones que presenta en términos de la superposición de las llamadas imágenes gemelas y orden cero (Goodman, 2005), lo cual dificultaba la observación de la imagen reconstruida. Se inventó entonces una configuración de holografía fuera de eje que alivió dicha superposición e impulsó el uso de la holografía (Leith and Upatnieks, 1964).
Mientras que el proceso de registro del holograma se puede modelar como una superposición en amplitud de las ondas objeto y referencia, el proceso de reconstrucción se puede entender como la difracción que una onda sufre en el holograma, entendido éste como una red difracción. Este entendimiento unido a la posibilidad de la manipulación digital de la información del holograma registrado (Goodman and Lawrence, 1967), abrió un nuevo espectro de oportunidades para la holografía y marcó la génesis de lo que hoy se conoce como holografía digital de la cual se reconoce su consolidación a mediados de los noventas (Schnars, 1994). El mundo digital permitió la visualización y cuantificación de la amplitud y la fase de la onda reconstruida y dotó la holografía de un conjunto nuevo de herramientas lo que significó un conjunto adicional de

\footnotetext{
Correspondencia:

Jorge Garcia-Sucerquia, jigarcia@unal.edu.co

Recibido: 13 de mayo de 2015

Aceptado: 21 de septiembre de 2015
} 
posibilidades por la versatilidad que brinda el mundo digital. Por ejemplo, fue posible remover las limitaciones que hacían la arquitectura en línea de Gabor una configuración poco atractiva, reviviendo el interés en esta simple, pero robusta arquitectura.

El mundo de la holografía digital eliminó la necesidad del procesado químico de las películas en las que se registraban los patrones de interferencia de los cuales se recuperaba la información del objeto de interés. El precio pagado por esta característica fue una reducida calidad de las imágenes recuperadas, plagadas de ruido de speckle (GarciaSucerquia, Ramírez, et al., 2006) y con la necesidad de magnificar los patrones de interferencia para posibilitar el grabado de los patrones de interferencia en los medios de registro digital disponibles en el mercado (Kreis, 2002). Se optó por configuraciones fuera de eje con reducidos ángulos entre las ondas de referencia y objeto (entre cero y tres grados) (Schnars, 1994; Picart and Leval, 2008), el uso de sistemas de magnificación de los patrones de interferencia (Cuche, Bevilacqua, and Depeursinge, 1999), o configuraciones en eje en las cuales la magnificación del patrón de interferencia se logra por la iluminación de la muestra con una onda esférica y la propagación en el espacio libre, tanto para electrones (Barton, 1988; Fink, et al., 1991) como para fotones (Kreuzer, et al., 1995; Jorge Garcia-Sucerquia, Xu, et al., 2006). Esta última opción fue la génesis de una técnica de microscopía sin lentes que inicialmente se conoció con el nombre de microscopía holográfica digital en línea (Garcia-Sucerquia, Xu, et al., 2006) y hoy se conoce como microscopía holográfica digital sin lentes (MHDSL). En este artículo se presentan los principios y algunos desarrollos de la MHDSL operando con múltiples longitudes de onda, desde la perspectiva de los aportes realizados por diversos grupos de trabajo coordinados por el autor del presente manuscrito.

\section{Microscopía Holográfica Digital Sin Lentes (MHDSL)}

La MHDSL es una metodología de formación de imágenes en dos etapas: i) el registro y, ii) la reconstrucción (GarciaSucerquia, Xu, et al., 2006; Jericho and Kreuzer, 2011). Para el registro la muestra es iluminada con ondas esféricas para garantizar la magnificación del patrón de difracción en el espacio libre y ser posible su registro en una cámara digital comercial. Para generar la onda esférica, una fuente de luz monocromática usualmente un láser de longitud de onda $\lambda$, es focalizada sobre un agujero con un diámetro del orden de $\lambda$. De esta forma la muestra, ubicada a una distancia $z$ del agujero, es iluminada por ondas esféricas altamente coherentes. La porción de onda débilmente esparcida por la muestra, conocida como onda objeto, interfiere sobre la superficie del sensor digital, ya sea una cámara CMOS (complementary metal-oxide semiconductor) o CCD (chargecoupled device), con la porción de la onda no esparcida, denominada onda de referencia. El sensor digital es posicionado de forma tal que su centro se encuentra a una distancia $L$ del agujero. La intensidad registrada por el sensor digital, llamada holograma en línea, se trasfiere a un PC para su procesamiento. En la Figura 1 se ilustra la etapa de registro de la MHDSL.

El holograma en línea se puede escribir como la superposición en amplitud de la onda objeto $U_{\text {scat }}(\mathrm{r})$ y la onda de referencia $U_{\text {ref }}(\mathrm{r})$ :

$I(\mathrm{r})=\left|U_{\text {scat }}(\mathrm{r})\right|^{2}+\left|U_{\text {ref }}(\mathrm{r})\right|^{2}+\left[U_{\text {scat }}(\mathrm{r}) U_{\text {ref }}^{*}(\mathrm{r})+U_{\text {scat }}^{*}(\mathrm{r}) U_{\text {ref }}(\mathrm{r})\right]$.

De acuerdo a la ecuación (1) la intensidad registrada por la cámara digital incluye los términos del orden cero de difracción $\left|U_{\text {scat }}(\mathrm{r})\right|^{2}+\left|U_{\text {ref }}(\mathrm{r})\right|^{2}$ los cuales hicieron impráctico el invento de Gabor (Gabor, 1948). La MHDSL resuelve esta dificultad por medio de la resta punto a punto de la irradiancia de la onda de referencia, correspondiente a la intensidad registrada sin la muestra presente, de la

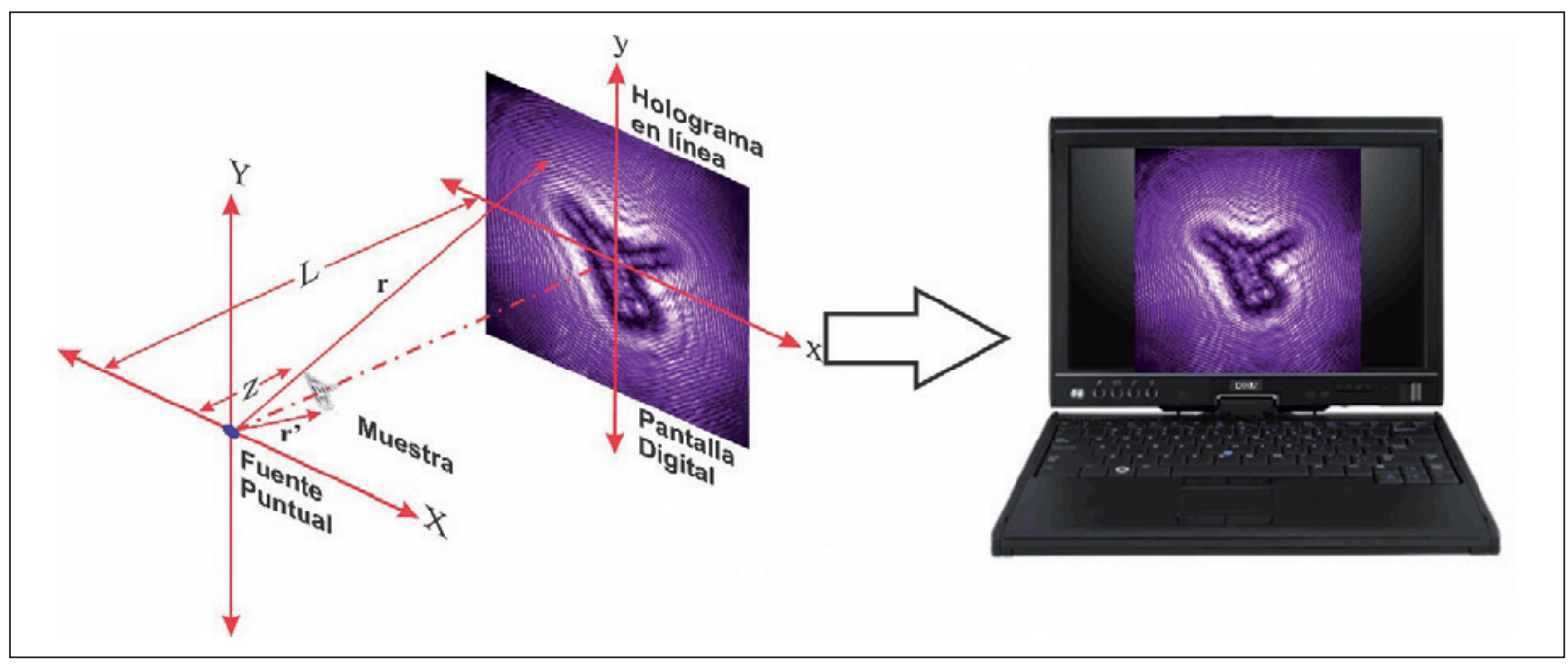

Figura 1. Etapa de registro en la microscopía holográfica digital sin lentes (MHDSL). 
intensidad presentada en la ecuación (1). Si adicionalmente se considera que la intensidad de la onda esparcida por el objeto es despreciable, se arriba a un nuevo holograma que denominamos holograma de contraste y tiene la forma:

$$
\tilde{I}(\mathrm{r})=\left[U_{\text {scat }}(\mathrm{r}) U_{\text {ref }}^{*}(\mathrm{r})+U_{\text {scat }}^{*}(\mathrm{r}) U_{\text {ref }}(\mathrm{r})\right] \text {. }
$$

Esta distribución de intensidad contiene la información de la onda objeto y la onda de referencia en su forma de imágenes gemelas (Goodman, 2005), las cuales no presentan problema alguno en el proceso de reconstrucción de los hologramas de contraste, como se ha demostrado en varias publicaciones (Garcia-Sucerquia, Xu, et al., 2006; Jericho and Kreuzer, 2011; Garcia-Sucerquia, 2013).

En la etapa de reconstrucción, la onda objeto, la cual porta la información de la muestra, es recuperada a partir del cálculo numérico del proceso de difracción que la onda conjugada de referencia sufre cuando ilumina el holograma de contraste. Ya que en MHDSL la onda de referencia es esférica, este proceso se puede describir matemáticamente por medio de la trasformada de Kirchhoff-Helmholtz (Barton, 1988; Garcia-Sucerquia, Xu, et al., 2006; Jericho and Kreuzer, 2011):

$$
U\left(\mathbf{r}^{\prime}\right)=\int_{\sigma} \tilde{I}(\mathbf{r}) \exp \left[i k\left(\frac{\mathbf{r}^{\prime} \cdot \mathbf{r}}{|\mathbf{r}|}\right)\right] d^{2} r
$$

En la ecuación (3) la integración se extiende sobre la superficie de la pantalla $\sigma$, cámara CCD o CMOS, con coordenadas $\mathrm{r}=$ $(x, y, L), k=2 \pi / \lambda$ es el número de onda; $\tilde{I}(\mathrm{r})$ es el holograma de contraste; y $\mathrm{r}^{\prime}=\left(x^{\prime}, y^{\prime}, z_{r}\right)$ son las coordenadas en el plano de reconstrucción. $U\left(r^{\prime}\right)$ es una cantidad compleja que puede ser calculada en varios planos de reconstrucción a diferentes distancias $z_{r}$ del agujero; esta opción permite recuperar la información tridimensional de la muestra o equivalentemente, la onda esparcida por el espécimen en diferentes planos. Esta operación se hace enteramente de forma numérica a partir de un holograma bidimensional de MHDSL con $M \times N$ pixeles. El holograma de contraste es interpolado en una malla tipo esférica con pixeles de tamaño $\Delta x^{\prime \prime}, \Delta y^{\prime \prime}$. Un nuevo holograma modificado $\tilde{I} "\left(m \Delta x^{\prime \prime}\right.$, $n \Delta y^{\prime \prime}$ ), el cual considera la distancia de propagación $z_{r}$, se introduce en la versión discreta de la ecuación (3) para producir (Kreuzer, 2002; Garcia-Sucerquia, Trujillo, and Restrepo Agudelo, 2014):

$$
\begin{aligned}
& U\left(s \Delta x^{\prime}, t \Delta y^{\prime}, z_{r}\right)= \\
& \Delta x^{\prime \prime} \Delta y^{\prime \prime} \exp \left[i k \frac{\left(s^{2} \Delta x^{\prime \prime} \Delta x^{\prime}+t^{2} \Delta y^{\prime \prime} \Delta y^{\prime}\right)}{2 L}\right] \\
& \quad \sum_{m=-M / 2 n=-N / 2}^{(M / 2)-1(N / 2)-1} \tilde{I}^{\prime \prime}\left(m \Delta x^{\prime \prime}, n \Delta y^{\prime \prime}\right) \\
& \\
& \times \exp \left[i k \frac{\left(m^{2} \Delta x^{\prime \prime} \Delta x^{\prime}+n^{2} \Delta y^{\prime \prime} \Delta y^{\prime}\right)}{2 L}\right] \\
& \times \exp \left[-i k \frac{\left((s-m)^{2} \Delta x^{\prime \prime} \Delta x^{\prime}+(t-n)^{2} \Delta y^{\prime \prime} \Delta y^{\prime}\right)}{2 L}\right]
\end{aligned}
$$

Según la ecuación (4), la amplitud de la onda objeto puede ser calculada como una convolución discreta por medio de tres transformadas rápidas de Fourier. En la ecuación (4) el tamaño de las coordenadas del plano de reconstrucción puede ser escogido independientemente de la distancia de reconstrucción, longitud de onda y tamaño del holograma, condición que es esencial en MHDSL para ajustar los tamaños de registro y reconstrucción. El proceso de reconstrucción aplicado al holograma que se envía al computador en la Figura 1, produce la imagen que se presenta en la Figura 2 (A). El perfil a lo largo de la línea recta sobre la reconstrucción del panel (A) se muestra en el panel (B). A partir de este perfil se puede observar que la técnica de MHDSL puede alcanzar resolución espacial del orden de los micrómetros, sin la necesidad de lentes. La calidad de la imagen reconstruida, permite distinguir claramente el empaquetamiento hexagonal de las esferas de poliestireno de $1.09 \mu \mathrm{m}$ de diámetro, que fueron usadas como muestra en este experimento.

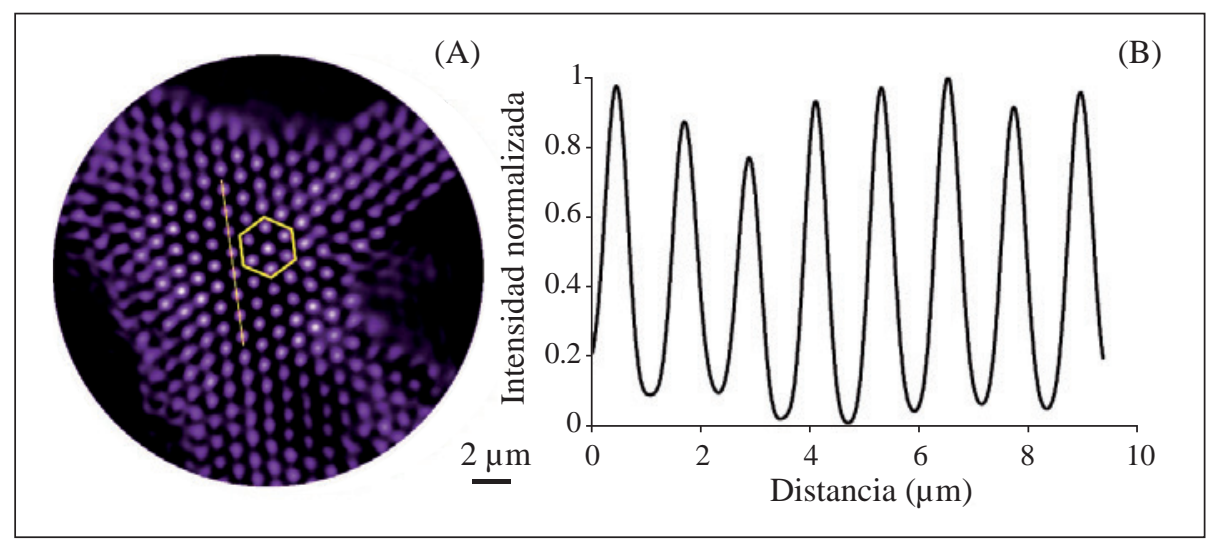

Figura 2. Reconstrucción (A) del holograma de contraste generado a partir del holograma en línea ilustrado en la Fig. 1. Perfil (B) a lo largo de la línea recta en el panel (A). 
Debido a su estructura, la ecuación (4) es totalmente paralelizable. Esta característica unida a la posibilidad del uso de unidades de procesamiento gráfico (GPU) para paralelizar cálculos de punto flotante, han permitido el computo de la ecuación (4) en GPUs de manera tal que la reconstrucción de un holograma de 2048x2048 pixeles se puede realizar en un computador portátil equipado con un tarjeta de video NVIDIA ${ }^{\circledR}$ Geforce $9800 \mathrm{GT}$ en $100 \mathrm{~ms}$ (Trujillo and Garcia-Sucerquia, 2013; Garcia-Sucerquia, Trujillo, and Restrepo Agudelo, 2014).

\section{MHDSL con fuentes multiespectrales}

La simplicidad, solidez y calidad del desempeño de MHDSL invita al desarrollo de arquitecturas de microscopía sin lentes que utilicen fuentes de iluminación multiespectrales para obtener información a diferentes longitudes de onda del espécimen en estudio. En este contexto, nuestro grupo de investigación ha sido pionero a nivel mundial en el desarrollo de la MHDSL a color (Garcia-Sucerquia, 2012) y su extensión a fuentes más complejas con espectros de emisión de cientos de nanómetros (Mendoza-Yero, Calabuig, et al., 2013; Mendoza-Yero, Tajahuerce, et al., 2013). La primera tecnología se ha denominado MHDSL a color y la segunda se enmarca el uso de láseres con pulsos ultra rápidos en MHDSL.

\section{a. MHDSL a color}

Para la generación de imágenes holográficas de especímenes microscópicos a color, en el montaje experimental de MHDSL ilustrado en la Figura 1 se incorporaron tres láseres con longitudes de onda en el rojo, verde y azul, respectivamente. Según se ilustra en la Figura 3 (A), la muestra se ilumina de forma secuencial por medio de los tres láseres para generar un holograma para cada una de las longitudes utilizadas. Las irradiancias de los láseres se ajustan de manera tal que operando de forma conjunta producen iluminación blanca sobre la muestra. El uso de los obturadores permite registrar de forma individual los hologramas en línea para el canal rojo, verde y azul; los hologramas registrados se ilustran en la Figura 3 (B), (C) y (D). Para el registro de los hologramas se utilizó, un microscopio sin lentes construido con una cámara CMOS monocromática de $1280 \times 1024$ pixeles cuadrados cada uno $6.7 \mu \mathrm{m}$ de lado. Se utilizó un microscopio con apertura numérica (NA) (Garcia-Sucerquia, Xu, et al., 2006) de 0.37 , es decir, para la cámara utilizada la distancia de la fuente puntal al centro de la misma fue $L=8.5 \mathrm{~mm}$. Tres láseres de estado sólido con longitudes de onda de $671 \mathrm{~nm}$ (rojo), 532 (verde), y 473nm (azul) fueron alineados por medio de los apropiados filtros dicroicos. Los tres haces se enfocaron sobre la superficie de un pinole con diámetro igual a 750nm por medio de un microscopio de objetivo apocromático. El uso de este objetivo, minimiza las variaciones de la cintura de los haces para las diferentes longitudes de onda de forma tal que la muestra es iluminada por un haz blanco para una perfecta iluminación. En este experimento se utilizó como muestra una sección de la cabeza de una mosca Drosophila melanogaster o mosca de la fruta. Esta muestra, con dimensiones de $250 \mu \mathrm{m}$ x $200 \mu \mathrm{m}$, posee un estructura interna compuesta por diferentes órganos y con variadas

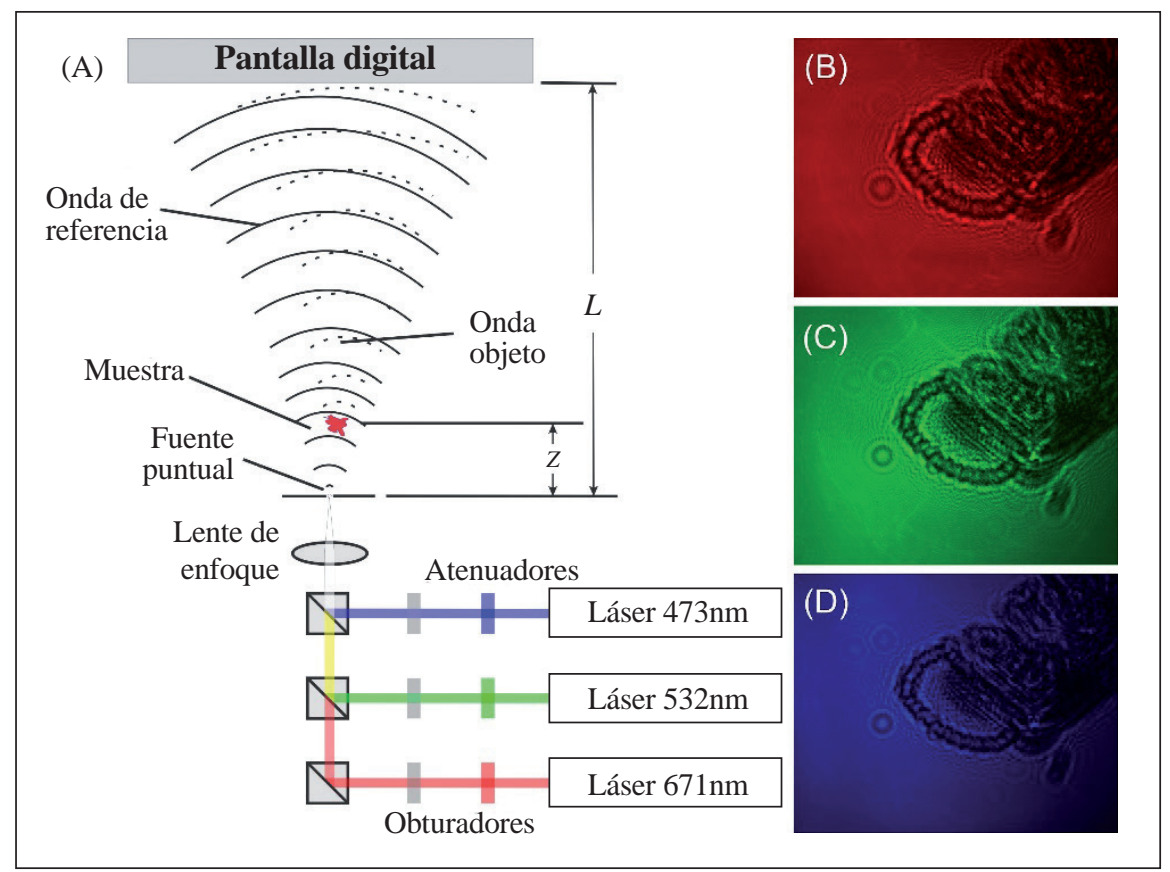

Figura 3. Ilustración de la etapa de registro en MHDSL a color. Panel (A) muestra el montaje experimental utilizado. Paneles (B), (C) y (D) corresponden a los hologramas registrados rojo, verde y azul, en este orden. 
respuestas espectrales, lo cual hace la sección de la cabeza de una mosca Drosophila melanogaster una muestra ideal para este estudio. La muestra fue preparada por medio de la técnica Bodian para fijar muestras por medio de parafina entre un par de placas de vidrio (Bodian, 1936).

La imagen reconstruida a color es obtenida por medio del procesamiento a posteriori de las reconstrucciones holográficas de los hologramas en línea para los canales rojo Figura 3 (B), verde (C) y azul (D). Cada holograma en línea a color es reconstruido con el uso de la apropiada longitud de onda insertada en la ecuación (4), asegurando que los hologramas reconstruidos tiene el mismo tamaño. Esta condición en los tamaños de los hologramas reconstruidos es de obligatorio cumplimiento para garantizar la mejor mezcla posible de las tres reconstrucciones holográficas correspondientes a cada canal de color. En los paneles (A), (B) y (C) de la Figura 4, se muestran las imágenes reconstruidas para los canales rojo, verde y azul, en este orden, obtenidos para una distancia de reconstrucción $\mathrm{z}=0.8 \mathrm{~mm}$. Debido a las homogeneidades de absorbancia para las tres longitudes de onda utilizadas, cada imagen reconstruida presenta detalles distintivos y/o diferentes niveles de irradiancia para las estructuras que constituyen la imagen de la muestra. En el panel (D) de la Figura 4, se muestra la imagen a color obtenida por medio del proceso de mezclado típico RGB de las imágenes en paneles (A), (B) y (C). La imagen a color exhibe detalles claramente visibles que son difícilmente observables en las imágenes monocromáticas. Esencialmente, el mezclado de la información adquirida con cada una de las longitudes de onda en una sola imagen a color deriva en un desempeño superior del MHDSL en comparación con su operación monocromática (Garcia-Sucerquia, Xu, et al., 2006). Esta mejora en la operación del MHDSL se origina en la recolección en una sola imagen de las diferentes vistas espectrales de la muestra.

Para evaluar la resolución espacial alcanzada por el MHDSL a color se analizó en gran detalle el panel (D) de la Figura 4. Esta figura es representada nuevamente en el panel (A) de la Figura 5, donde se resaltan detalles con tamaños superiores a los 3 micrométros que son visibles en la imagen reconstruida a color. En esta imagen el conjunto de omatidias, estructura rojiza en el borde del ojo compuesto de la mosca de la fruta, son observables claramente. Para un análisis más cuantitativo, se muestran en el panel (B) por medio de una línea sólida los perfiles transversales de dos antenas situadas entre las omatidias. Dicha línea sólida corresponde al perfil de intensidad a lo largo de la línea blanca trazada al interior del círculo blanco del panel (A). Estas secciones transversales muestran la capacidad de MHDSL a color de formar imágenes incluso de estructuras sub-micrométricas. Otro detalle de interés es resaltado en el área cuadrada del panel (A). En esta área se recogen tres omatidias que son magnificadas en la esquina superior derecha de este panel. La línea punteada en el panel (B)
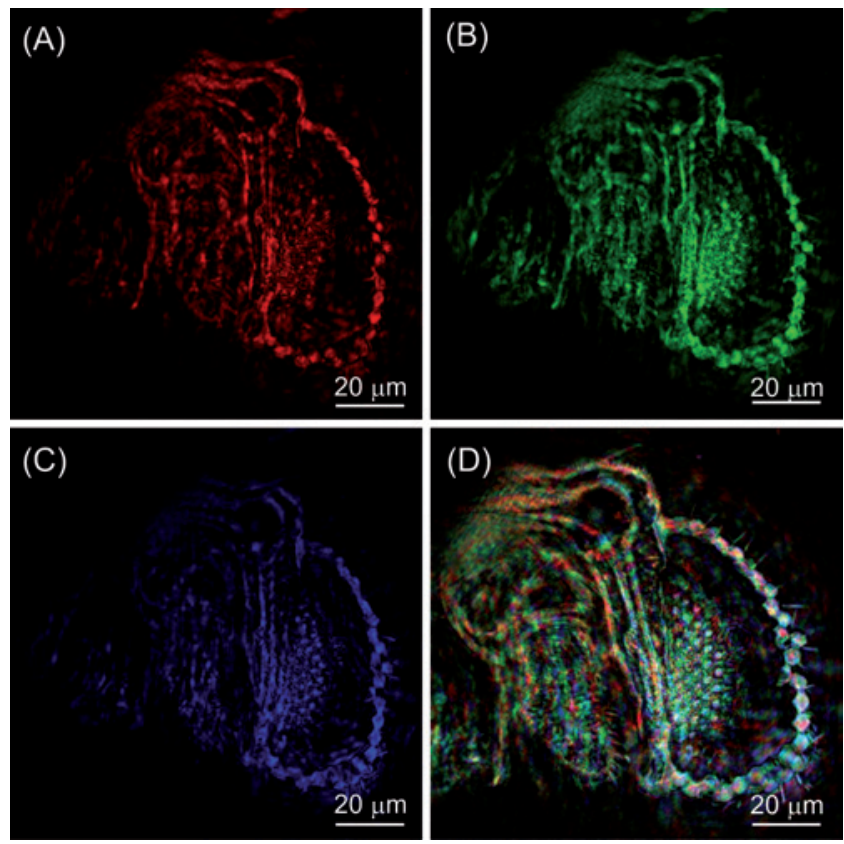

Figura 4. Reconstrucción de imágenes a color en MHDSL. Paneles de (A) hasta (C) son las imágenes reconstruidas para los canales individuales de los colores rojo, verde y azul, en este orden. En el panel (D) se muestra la imagen a color resultado de la composición de las imágenes reconstruidas en los paneles (A), (B) y (C).

representa el perfil a lo largo de la estructura de tres puntos situados entre el omatidias. Esta línea punteada muestra la capacidad de la MHDSL a color de formar imágenes de estructuras a nivel micrométrico a color alcanzando resolución lateral del orden de los $2 \mu \mathrm{m}$.

\section{b. FEMTO Laser MHDSL}

Los resultados alcanzados con la MHDSL a color invitan a extender el uso de esta tecnología de microscopía con fuentes de iluminación con ancho espectral continuo en el rango de cientos de nanómetros. Fuentes que presentan estas características y son gran interés en la actualidad son los láseres ultra rápidos. Estas fuentes tienen la característica particular de presentar una considerable coherencia espacial pero despreciable coherencia temporal, lo que determina su desempeño en cualquier metodología de formación de imágenes. Debido a esta característica su uso en sistemas de microscopía holográfica digital impone el uso de arreglos de redes de difracción para manipular los frentes de onda y obtener ondas de iluminación con las condiciones de coherencia espacial y temporal que demanda la microscopía holográfica digital (Witte, et al., 2012). Gracias a su arquitectura en eje, la longitud de coherencia temporal de las fuentes requeridas en MHDSL está en el orden de los micrómetros, lo que ha posibilitado el uso sistemas de iluminación tipo LED en esta metodología de microscopía (Repetto, Piano, and Pontiggia, 2004; Garcia-Sucerquia, 2013; Petruck, Riesenberg, and 

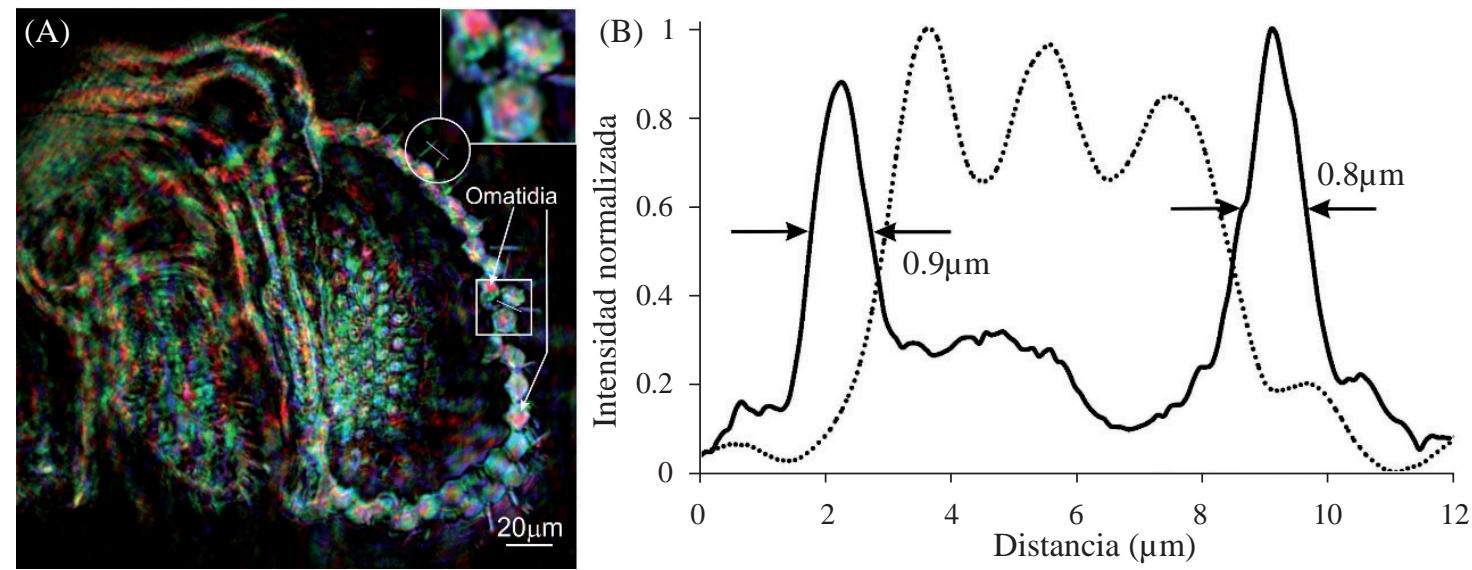

Figura 5. Imagen reconstruida a color con resolución micrométrica. (A) muestra la imagen reconstruida a color de una sección de la cabeza de la mosca de la fruta registrada con $N A=0.37(L=8.5 \mathrm{~mm})$. Las omatidias rojizas típicas de la mosca de la fruta salvaje se observan claramente. Las líneas sólidas/punteadas en el panel (B) representan el perfil a lo largo de la línea blanca en el círculo/cuadrado del panel (A). El área cuadrada es magnificada en la esquina del panel (A).

Kowarschik, 2012) y por ende sugiere a la utilización de fuentes más complejas con esta misma característica como los láseres ultra rápidos (Rosenhahn, et al., 2009; Brunel, et al., 2012; Mendoza-Yero, Calabuig, et al., 2013). Nuestro grupo de investigación fue pionero en el mundo en la utilización de láseres de femto segundo en la visualización de muestras biológicas por medio de la MHDSL (MendozaYero, Calabuig, et al., 2013). Para contrastar los resultados obtenidos por esta técnica, se desarrolló un MHDSL operando de forma simultánea con un láser mode-locked Ti:Sa (12 fs@ FWHM, 800 nm longitud de onda media, $4 \mathrm{~nJ}$ energía por pulso y $75 \mathrm{MHz}$ tasa de repetición) y una LED con longitud de onda media $655 \pm 20 \mathrm{~nm}$; el montaje es ilustrado en la Figura 6.

Como se ha mencionado anteriormente, en MHDSL la fuente de iluminación de la muestra es generada focalizando luz en un pinhole de diámetro del orden de la longitud de onda $d_{p} \approx$ $\lambda$, es decir, la luz es empaquetada en un área del orden $\pi \lambda^{2} / 4$. Este confinamiento de la radiación electromagnética impone que por ejemplo para un láser típico de $\mathrm{He}-\mathrm{Ne}$ laser de 10 $\mathrm{mW}$, la potencia por unidad de área sobre la superficie del pinhole es del orden de $0.7 \mathrm{MW} / \mathrm{cm}^{2}$. Esta cifra está en el rango de seguridad para no dañar los pinholes comerciales los cuales pueden manipular irradiancias del orden de 70 $\mathrm{TW} / \mathrm{cm}^{2}$. Sin embargo, en nuestra aplicación con láseres ultra rápidos la potencia pico sobre la superficie del pinhole es del orden de $2.5 \mathrm{TW} / \mathrm{cm}^{2}$, lo cual sugiere una reducción de la irradiancia por medio de filtros neutros; en este experimento dicha potencia se redujo a $1.1 \mathrm{TW} / \mathrm{cm}^{2}$.

Para ambas fuentes de iluminación se utilizaron pinholes de 5 y $1 \mu \mathrm{m}$ de diámetro sobre los cuales se focalizó la luz de la fuente respectiva por medio de un objetivo de microscopio M-40X producido por Newport. Este objetivo tiene una apertura numérica de 0.65 , distancia de trabajo de $0.6 \mathrm{~mm}$ y

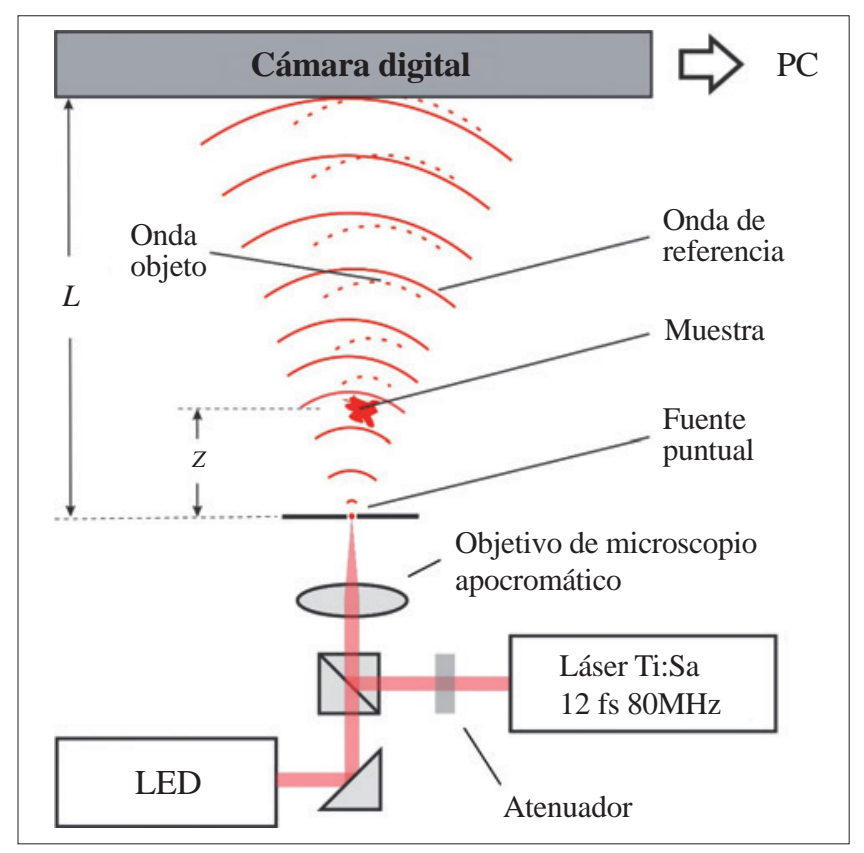

Figura 6. Esquema de un MHDSL usando simultáneamente una láser Ti:Sa y un LED.

distancia focal efectiva $F=4.5 \mathrm{~mm}$. Con esta configuración el diámetro más pequeño del spot sobre el pinhole fue de $1.2 \mu \mathrm{m}$; el más grande fue $2 \mu \mathrm{m}$. Los hologramas en línea se registraron en una cámara CCD Balser ${ }^{\circledR}$ con 1024x1024 pixeles cuadrados de $6 \mu \mathrm{m}$ de lado. La cámara se ubicó a una distancia entre 5 y $17 \mathrm{~mm}$ desde el pinhole.

Una sección de la cabeza de una mosca Drosophila melanogaster se utilizó como muestra para este estudio. Las reconstrucciones de los hologramas para esta muestra biológica de compleja estructura interna, para ambos 
fs-MHDSL y LED-MHDSL, se muestran en la Figura 7. Para todos los paneles de la figura no se observa ruido coherente, como se espera de los trabajo previos (Petruck, Riesenberg, and Kowarschik, 2012; Garcia-Sucerquia, 2013), debido a la reducida longitud de coherencia del láser de femto segundos. Cuando se comparan los paneles de la fila superior con los de la fila inferior, se puede concluir que la reducción del ruido coherente que se logra con ambas fuentes es comparable. Sin embargo, hay una diferencia importante en la resolución espacial de los hologramas reconstruidos. Se ha reportado que la resolución espacial alcanzable en MHHDSL se mejora con la reducción del tamaño del pinhole, esencialmente porque la coherencia espacial de la fuente de iluminación sobre la muestra es incrementada (Garcia-Sucerquia, Xu, et al., 2006). De los paneles (C) y (D) esta premisa se puede validar, pero para los paneles (A) y (B) ésta no es cierta.

Para entender el desempeño de la MHDSL operando con el láser de femto segundos debemos profundizar un poco en la operación del microscopio. La borrosidad que aparece en las imágenes reconstruidas para el fs-MHDSL puede ser atribuida al ancho espectral del láser. En este contexto, el láser de femto segundos se puede entender como una fuente constituida por múltiples subfuentes con diferentes
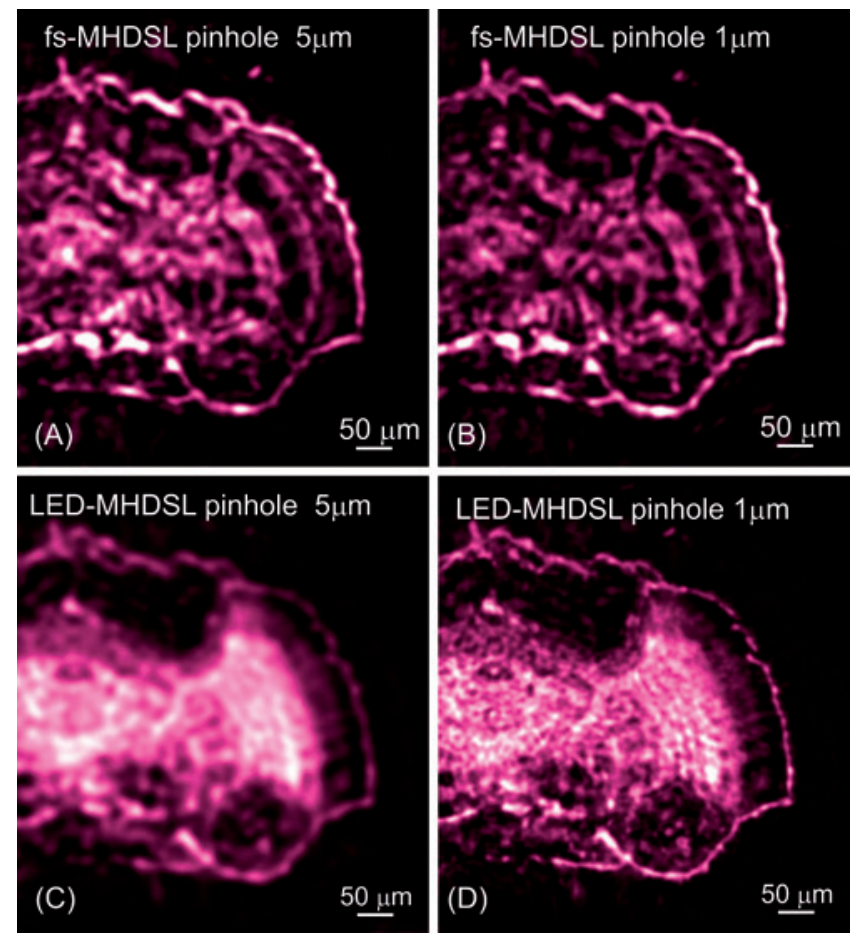

Figura 7. Reconstrucción de los hologramas para fs-MHDSL y LED-MHDSL. Paneles (A) y (B) muestran las reconstrucciones para fs-MHDSL operando con pinholes de 5 y $1 \mu \mathrm{m}$ de diámetro, respectivamente. Paneles (C) y (D) son las reconstrucciones de los hologramas para LED-MHDSL usando pinholes de diámetro de 5 and $1 \mu \mathrm{m}$, en ese orden. longitudes de onda y mutuamente incoherentes. De esta forma el holograma en línea estará dado por la superposición incoherente de múltiples hologramas monocromáticos en línea cada uno con la forma:

$$
\begin{aligned}
I^{\prime}(\mathrm{r}, \lambda) & =\left|U_{\text {scat }}(\mathrm{r}, \lambda)\right|^{2}+\left|U_{\text {ref }}(\mathrm{r}, \lambda)\right|^{2} \\
& +\left[U_{\text {scat }}(\mathrm{r}, \lambda) U_{\text {ref }}^{*}(\mathrm{r}, \lambda)+U_{\text {scat }}^{*}(\mathrm{r}, \lambda) U_{\text {ref }}(\mathrm{r}, \lambda)\right] .
\end{aligned}
$$

De forma similar como se mencionó para la ecuación (1), en la ecuación (5) $\left|U_{\text {scat }}(\mathrm{r}, \lambda)\right|^{2}$ es despreciable debido a que las muestras utilizadas en MHDSL esparcen muy débilmente la onda que las ilumina. Los términos entre los corchetes, en la ecuación 5, son las imágenes gemelas, las cuales no perturban la reconstrucción del holograma, como ha sido ampliamente estudiado en otras referencias (GarciaSucerquia, Xu, et al., 2006; Garcia-Sucerquia, 2013).

Cada uno de los hologramas en línea estará sopesado por el valor de la potencia espectral $\rho(\lambda)$ de la fuente para cada longitud de onda, de forma tal que el holograma en línea registrado estará dado por:

$$
I(\mathbf{r})=\int_{\lambda_{0}-\Delta \lambda / 2}^{\lambda_{0}+\Delta \lambda / 2} \rho(\lambda) I^{\prime}(\mathbf{r}, \lambda) d \lambda
$$

$\operatorname{con} \lambda_{0}$ la longitud de onda promedio y $\Delta \lambda$ el ancho espectral la fuente de iluminación. Debido a que el tiempo de integración de la cámara es mucho más grande que ancho temporal de los pulsos del láser (Gu, 2000), para este experimento en particular el holograma de contraste tendrá la forma:

$$
\tilde{I}(\mathbf{r})=\int_{\lambda_{0}-\Delta \lambda / 2}^{\lambda_{0}+\Delta \lambda / 2} \rho(\lambda)\left[I^{\prime}(\mathbf{r}, \lambda)-\left|U_{r e f}(\mathbf{r}, \lambda)\right|^{2}\right] d \lambda
$$

La amplitud compleja de la onda esparcida por el objeto $U_{\text {scat }}(\mathrm{r}, \lambda)$ para cada longitud de onda puede ser computada por medio de la difracción de la onda de referencia $U_{\text {ref }}(r$, ג) cuando ilumina el holograma de contraste siguiendo lo expresado en la ecuación (3).

Cuando el holograma de contraste es representado por la ecuación (7), la información de las longitudes de onda individuales es mezclada, de forma tal que la longitud de onda utilizada en la reconstrucción cambia la escala de la misma. De esta forma la onda objeto recuperada $U_{\text {scat }}(\mathrm{r})$ corresponde a la superposición incoherente de múltiples campos difractados del espécimen cada uno con una escala diferente proporcional a $\lambda / \lambda_{0}$; esta superposición es la responsable de las imágenes borrosas que se muestran en la Figura 7 (A) y (B).

La explicación de la invariancia de la resolución especial del fs-MHDSL con la variación del tamaño de pinole se puede soportar en el entendimiento del MHDSL como un microscopio interferométrico de división de frente de onda. En este contexto, únicamente la coherencia espacial de la fuente determina el desempeño del microscopio y puesto que el láser de femto segundos es espacialmente completamente coherente, los diferentes pinholes no modifican la coherencia 
espacial sobre el plano de la muestra y por ende no se puede observar un cambio en el desempeño del microscopio. Para fuentes con coherencia espacial parcial como los LEDs, el diámetro del área que es iluminada coherentemente $D_{\text {coh }}$ en un plano localizado a una distancia $Z$ del pinole de diámetro $d_{p}$ está dada por el teorema de Van Cittert-Zernike theorem, $D_{c o h} \approx 0.32 \lambda_{0} Z / d_{p}$. Esta expresión indica que a menor diámetro de pinhole mayor área de coherencia y por ende mejor resolución espacial del MHDSL (Petruck, Riesenberg, and Kowarschik, 2012; Garcia-Sucerquia, 2013). Esta característica se pude verificar en la Figura 7 paneles (C) y (D). Debido a que el láser de femto segundos es complemente coherente espacialmente, el desempeño del fs-MHDSL no es modificado por el tamaño del pinhole, como se muestra en la Figura 7 (A) y (B). La borrosidad de las imágenes reconstruidas se debe desde luego a la superposición incoherente de las múltiples imágenes reconstruidas con diferentes escalas, según se mencionó anteriormente.

\section{Conclusiones}

Se ha presentado en este trabajo una recopilación de los aportes más destacados realizados por nuestro grupo de investigación en el dominio de la MHDSL operando con fuentes de iluminación de múltiple longitud de onda y alcanzado resolución micrométrica.

Se ilustró como por primera vez se desarrolló una estrategia para observar muestras microscópicas a color y sin el uso de lentes por medio de la MHDSL, donde se logró la visualización de estructuras submicrométricas y resolución espacial en el orden de los 2 micrómetros.

Se reportó el uso de fuentes de iluminación con ancho espectral continuo en el rango de los cientos de nanométros en la arquitectura MHDSL. Se mostró como su uso para visualizar muestras biológicas con estructura interna, reveló el papel de la coherencia espacial y temporal de la fuente de iluminación en el desempeño del MHDSL. El análisis de la incidencia de la coherencia espacial y temporal en la MHDSL se llevó a cabo por medio de la comparación de los resultados obtenidos usando iluminación tipo LED y láser de femto segundos.

\section{Agradecimientos}

El autor agradece el soporte de la Universidad Nacional de Colombia, Proyecto Hermes 19384, el Programa de Internacionalización del Conocimiento y el programa de Jóvenes Investigadores de Colciencias-Universidad Nacional de Colombia código Hermes 28751.

\section{Conflicto de intereses}

El autor declara que no tiene conflicto de intereses.

\section{Referencias}

Barton, J. J. (1988). Photoelectron Holography. Physical Review Letters, 61 (12). American Physical Society, 1356-59.
Bodian, D. (1936). A New Method for Staining Nerve Fibers and Nerve Endings in Mounted Paraffin Sections. The Anatomical Record, 65 (1). Wiley Subscription Services, Inc., A Wiley Company, 89-97.

Brunel, M., Shen, H., Coetmellec, S., Lebrun, D., and Ait Ameur, H. (2012). Femtosecond Digital in-Line Holography with the Fractional Fourier Transform: Application to PhaseContrast Metrology. Applied Physics B, 106 (3). SpringerVerlag, 583-91.

Cuche, E., Bevilacqua, H., and Depeursinge, C. (1999). Digital Holography for Quantitative Phase-Contrast Imaging. Opt. Lett., 24 (5). OSA, 291-93.

Fink, H-W., Schmid, H., Kreuzer, H., and Wierzbicki, A. (1991). Atomic Resolution in Lensless Low-Energy Electron Holography. Physical Review Letters, 67 (12): 1543-46.

Gabor, D. (1948). A New Microscopic Principle. Nature, 161: 777-78.

Gabor, D. (1949). Microscopy by Reconstructed Wave-Fronts. Proc. R. Soc. London A, 197: 454.

Gabor, D. (1951). Microscopy by Reconstructed Wave Fronts: II. Proc. Phys. Soc. London B, 64: 449.

Garcia-Sucerquia, J., Trujillo, C., and Restrepo, J. (2014). Microscopio Holográfico Digital Sin Lentes (MHDSL) y Método Para Visualizar Muestras. Colombia: SIC (Colombia).

Garcia-Sucerquia, J. (2012). Color Lensless Digital Holographic Microscopy with Micrometer Resolution. Optics Letters, 37 (10): 1724-26.

Garcia-Sucerquia, J. (2013). Noise Reduction in Digital Lensless Holographic Microscopy by Engineering the Light from a Light-Emitting Diode. Applied Optics, 52 (1): A232-39.

Garcia-Sucerquia, J., Herrera-Ramírez, J., Castaneda, R. (2006). Incoherent Recovering of the Spatial Resolution in Digital Holography. Optics Communications, 260 (1): 62-67.

Garcia-Sucerquia, J., Xu, W., Jericho, S., Klages, P., Jericho, M., and Kreuzer, H. (2006). Digital in-Line Holographic Microscopy. Appl. Opt., 45 (5). OSA, 836-50.

Goodman, J W, and Lawrence, L. (1967). Digital image formation from electronically detected holograms. Applied Physics Letters, 11 (3).

Goodman, J W. (2005). Introduction to Fourier Optics. Greenwood Village: Roberst \& Company Publishers.

Gu, M. (2000). Advanced Optical Imaging Theory. Springer Series in Optical Sciences,. Vol. 75. Springer.

Jericho, M H, and Kreuzer, H. (2011). Point Source Digital In-Line Holographic Microscopy. In Coherent Light Microscopy, edited by P Ferraro, A Wax, and Z Zalevvsky, 3-30. Springer-Verlag Berlin Heidelberg.

Kreis, T. (2002). Frequency Analysis of Digital Holography. Optical Engineering, 41 (4): 771-78.

Kreuzer, H. (2002). "Holographic microscope and method of hologram reconstruction,” US,6411406. 
Kreuzer, H J, Fink, H., Schmid, H., and Bonev, S. (1995), Holography of Holes, with Electrons and Photons. Journal of Microscopy, 178 (3), 191-97.

Leith, N., and Upatnieks, J. (1964). Wavefront Reconstruction with Diffused Illumination and Three-Dimensional Objects. Journal of the Optical Society of America, 54 (11). OSA, 1295-1301.

Mendoza-Yero, O., Calabuig, A., Tajahuerce, E., Lancis, J., Andrés, P., and Garcia-Sucerquia, J. (2013). Femtosecond Digital Lensless Holographic Microscopy to Image Biological Samples. Optics Letters, 38 (17). OSA, 3205-7.

Mendoza-Yero, O., Tajahuerce, E., Lancis, J., and GarciaSucerquia, J. (2013). Diffractive Digital Lensless Holographic Microscopy with Fine Spectral Tuning. Optics Letters, 38 (12): 2107-9.

Petruck, P, Riesenberg, R., and Kowarschik, R. (2012). Optimized Coherence Parameters for High-Resolution Holographic Microscopy. Applied Physics B, 106 (2). Springer-Verlag, 339-48.

Picart, P., and Leval, J. (2008). General Theoretical Formulation of Image Formation in Digital Fresnel Holography: Erratum. J. Opt. Soc. Am. A, 26 (2). OSA, 244.
Repetto, L, Piano, E., and Pontiggia, C. (2004). Lensless Digital Holographic Microscope with Light-Emitting Diode Illumination. Opt. Lett., 29 (10). OSA, 1132-34.

Rosenhahn, A., Staier, F., Nisius, T., Schäfer, D., Barth, R., Christophis, C., Stadler, M. et al. (2009). Digital In-Line Holography with Femtosecond VUV Radiation Provided by the Free-Electron Laser FLASH. Opt. Express, 17 (10). OSA, 8220-28.

Schnars, U. (1994). Direct Phase Determination in Hologram Interferometry with Use of Digitally Recorded Holograms. J. Opt. Soc. Am. A, 11 (7). OSA, 2011-15.

Trujillo, C, and Garcia-Sucerquia, J. (2013). Accelerated Numerical Processing of Electronically Recorded Holograms With Reduced Speckle Noise. Image Processing, IEEE Transactions on, 22 (9): 3528-37.

Witte, S., Plauska, A., Ridder,M., Berge, Huibert D Mansvelder, H., and Groot, M. (2012). Short-Coherence off-Axis Holographic Phase Microscopy of Live Cell Dynamics. Biomed. Opt. Express, 3 (9). OSA, 2184-89. 